\title{
Progressive Tricuspid Regurgitation and Elevated Tricuspid Regurgitation Pressure Gradient after Transvenous Permanent Pacemaker Implantation
}

\author{
Wei-Chieh Lee ${ }^{1}$, Hsiu-Yu Fang ${ }^{1}$, Huang-Chung Chen ${ }^{1}$, Yung-Lung Chen ${ }^{1}$, Tzu-Hsien Tsai ${ }^{1}$, \\ Kuo-Li Pan ${ }^{1}$, Yu-Sheng Lin ${ }^{1}$, Wen-Hao Liu ${ }^{1}$, and Mien-Cheng Chen ${ }^{2}$ \\ ${ }^{1}$ Chang Gung Memorial Hospital Kaohsiung Branch \\ ${ }^{2}$ Chang Gung Memorial Hospital-Kaohsiung Medical Center, Chang Gung University \\ College of Medicine
}

June 25, 2020

\begin{abstract}
Background The association of post-implant tricuspid regurgitation (TR) and heart failure (HF) hospitalization in patients without HF and preexisting abnormal TR and TR pressure gradient (PG) remain unclear. This study aimed to explore the clinical outcomes about progressive post-implant TR after permanent pacemaker (PPM) implantation. Methods A total of 1,670 patients who underwent a single ventricular or dual-chamber transvenous PPM implantation at our hospital between January 2003 and December 2017 were included in the study. Patients with prior valvular surgery, heart failure (HF), and baseline abnormal TR and TRPG were excluded. Finally, a total of 1,075 patients were enrolled in this study. Progressive TR was defined as increased TR grade of [?]2 degrees and/or TRPG of $>30$ mmHg after implant. Results 198 (18.4\%) patients (group 1) experienced progressive post-implant TR and/or elevated TRPG. Group 1 had 1 larger changes in post-implant TRPG (group 1 vs. group $2 ; 12.8 \pm 9.6 \mathrm{mmHg}$ vs. $1.1 \pm 7.6 \mathrm{mmHg} ; \mathrm{p}<0.001)$ than group 2 without progressive post-implant TR. Group 1 had a higher incidence of HF hospitalization compared to group 2 (13.6\% vs. 4.7\%; p < 0.001). Pre-implant TRPG (HR: 1.075 ; 95\% confidence interval $(\mathrm{CI}): 1.032-1.121 ; \mathrm{p}=0.001$ ) and post-implant left atrial dimension (HR: 1.076; 95\% CI: 1.038-1.114; $\mathrm{p}$ $<0.001$ ) were independent predictors of progressive post-implant TR. Conclusion After a transvenous ventricular-based PPM implantation, $18.4 \%$ of patients experienced progressive post-implant TR and/or elevated TRPG. Higher pre-implant TRPG and larger post-implant LA dimension were independent predictors of progressive post-implant TR.
\end{abstract}

Progressive Tricuspid Regurgitation and Elevated Tricuspid Regurgitation Pressure Gradient after Transvenous Permanent Pacemaker Implantation

Wei-Chieh Lee, MD ${ }^{\mathrm{a}, \mathrm{b}}$, Hsiu-Yu Fang, MD ${ }^{\mathrm{b}}$, Huang-Chung Chen, MD ${ }^{\mathrm{b}}$, Yung-Lung Chen, MD ${ }^{\mathrm{b}}$, Tzu-Hsien Tsai, MD ${ }^{b}$, Kuo-Li Pan, MD ${ }^{c}$, Yu-Sheng Lin, MD ${ }^{c}$, Wen-Hao Liu, MD ${ }^{b}$, Mien-Cheng Chen, MD ${ }^{b}$

${ }^{a}$ Institute of Clinical Medicine, College of Medicine, National Cheng Kung University, Tainan, Taiwan

${ }^{\mathrm{b}}$ Division of Cardiology, Department of Internal Medicine, Kaohsiung Chang Gung Memorial Hospital, Chang Gung University College of Medicine, Kaohsiung, Taiwan

${ }^{\mathrm{c}}$ Division of Cardiology, Chang Gung Memorial Hospital, Chiayi, Taiwan

Address for correspondence: Mien-Cheng Chen, MD

Division of Cardiology, Department of Internal Medicine, Kaohsiung Chang Gung Memorial Hospital, Chang Gung University College of Medicine, 123 Ta Pei Road, Niao Sung District, Kaohsiung City, 83301, Taiwan 
Tel: 886-7-731-7123 ext. 8300

Fax: 886-7-732-2402

E-mail: chenmien@ms76.hinet.net

Running title: Progressive tricuspid regurgitation after pacemaker implantation

Word count: 3387

Sources of funding

None

Disclosures

None

\section{Conflicts of interest}

All authors declare to have no conflicts of interest.

\section{Human rights statements and informed consent}

All procedures followed were in accordance with the ethical standards of the responsible committee on human experimentation (institutional and national) and with the 1964 Helsinki Declaration and its later revisions.

\section{Abstract}

\section{Background}

The association of post-implant tricuspid regurgitation (TR) and heart failure (HF) hospitalization in patients without $\mathrm{HF}$ and preexisting abnormal TR and TR pressure gradient (PG) remain unclear. This study aimed to explore the clinical outcomes about progressive post-implant TR after permanent pacemaker (PPM) implantation.

\section{Methods}

A total of 1,670 patients who underwent a single ventricular or dual-chamber transvenous PPM implantation at our hospital between January 2003 and December 2017 were included in the study. Patients with prior valvular surgery, heart failure (HF), and baseline abnormal TR and TRPG were excluded. Finally, a total of 1,075 patients were enrolled in this study. Progressive TR was defined as increased TR grade of [?]2 degrees and/or TRPG of $>30 \mathrm{mmHg}$ after implant.

\section{Results}

198 (18.4\%) patients (group 1) experienced progressive post-implant TR and/or elevated TRPG. Group 1 had 1 larger changes in post-implant TRPG (group 1 vs. group 2; $12.8 \pm 9.6 \mathrm{mmHg}$ vs. $1.1 \pm 7.6 \mathrm{mmHg}$; $\mathrm{p}<0.001)$ than group 2 without progressive post-implant TR. Group 1 had a higher incidence of HF hospitalization compared to group 2 ( $13.6 \%$ vs. $4.7 \%$; p < 0.001). Pre-implant TRPG (HR: 1.075; $95 \%$ confidence interval $(\mathrm{CI}): 1.032-1.121 ; \mathrm{p}=0.001)$ and post-implant left atrial dimension (HR: $1.076 ; 95 \% \mathrm{CI}$ : 1.038-1.114; $\mathrm{p}<0.001)$ were independent predictors of progressive post-implant TR.

\section{Conclusion}

After a transvenous ventricular-based PPM implantation, $18.4 \%$ of patients experienced progressive postimplant TR and/or elevated TRPG. Higher pre-implant TRPG and larger post-implant LA dimension were independent predictors of progressive post-implant TR.

Keywords: Tricuspid regurgitation; Transvenous permanent pacemaker implantation; Heart failure hospitalization

\section{Background}


In 1959, an endocardial transvenous lead was firstly introduced for permanent cardiac pacing, which has great benefits in reducing cardiac morbidity and mortality related to symptomatic bradycardia ${ }^{1,2}$. However, the introduction of transvenous right ventricular pacing leads across the tricuspid valve can be associated with the development of tricuspid regurgitation (TR) and elevated tricuspid regurgitation pressure gradient (TRPG). Indeed, the prevalence of TR was increased in patients with transvenous permanent pacemaker (PPM) compared with the general population ${ }^{3}$. One previous report demonstrated that $21.2 \%$ of patients developed worsening TR degree after the transvenous lead implantation and a higher rate of worsening TR in patients with implantable cardioverter defibrillator (ICD) lead compared with $\mathrm{PPM}^{4}$. Another study showed that device type and number of leads placed did not affect the worsening degree of post-implant $\mathrm{TR}^{5}$.

The underlying mechanisms of transvenous cardiac pacing-related TR is not fully understood. Several mechanisms have been proposed that included a mechanical effect of the lead interfering the motion of the tricuspid leaflets, RV pacing-induced desynchronization ${ }^{6,7}$ and leads related tricuspid leaflet injury or perforation, entanglement, impingement, or adherence to the tricuspid valve ${ }^{6}$. One study reported that worsening TR occurred only in the chronic phase over 2 years, whereas another study reported a temporal trend toward increasing TR both acutely and chronically over 4 years after cardiac devices implantation ${ }^{5,8}$. Therefore, the prevalence of increased degree of post-implant TR remains conflicting. Moreover, the association of postimplant TR and heart failure (HF) hospitalization in patients without HF and preexisting abnormal TR and abnormal TRPG remains unclear. Accordingly, we conducted this study to assess the prevalence of TR after cardiac device implantation and determine its clinical significance on HF hospitalization in a large retrospective cohort after transvenous ventricular-based PPM implantation.

\section{Methods}

\section{Patient population}

A total of 1,670 patients who underwent a single ventricular or dual-chamber transvenous PPM implantation at our hospital between January 2003 and December 2017 were included in this study. Patients with prior valvular surgery, HF and left ventricular ejection fraction (LVEF) $<50 \%$, dilated cardiomyopathy, hypertrophic cardiomyopathy, and preexisting abnormal (mild-moderate, moderate or severe) TR and abnormal $(>30$ $\mathrm{mmHg}$ ) TRPG were excluded. Patients without follow-up records for PPM and without complete follow-up echocardiography were also excluded (Figure 1). Finally, a total of 1,075 patients were enrolled in this study and were divided into two groups: group 1 consisted of 198 patients with increased degree of post-implant TR ([?]2 degrees) and/or abnormal TRPG and group 2 consisted of 877 patients without increased degree of post-implant TR and abnormal TRPG. Patients with dual-chamber PPM implantation underwent pacing in the dual chamber rate-adaptive mode, whereas patients with single ventricular PPM implantation underwent pacing in the ventricular-inhibited rate-adaptive mode. General demographics, comorbidities, lead positions, pacing QRS durations, pacing percentages, echocardiographic parameters, HF hospitalization, and cardiovascular and all-cause mortality were compared between the groups.

\section{Follow-up}

Baseline electrocardiographic (ECG) and echocardiographic parameters were acquired from the ECG and echocardiography performed nearest to the implant date. Pacing-lead locations were reviewed using anteroposterior, right-oblique, and left-oblique views after implantation. PPM records were obtained at regular intervals (at least 6 months), and the ventricular pacing burden (ventricular pacing percentage) was obtained by telemetry at the follow-up. The pacing QRS duration was measured within 3 days after PPM implantation from the surface 12-lead ECG. Patients visited the outpatient department at regular intervals (3-6 months).

\section{Ethical statement}

This study conformed to the ethical guidelines of the 1975 Declaration of Helsinki and was approved for human research by the institutional review committee of Hospital.

\section{Echocardiography}


Echocardiographic parameters, including left atrial (LA) dimension, LVEF, LV end-diastolic volume (LVEDV), and TR grade/TRPG, were measured using GE Vivid 9 or Philips IE33 acccording to the recommendations from the american society of echocardiography. LVEF and LVEDV were quantified by the M-mode and corrected by the two-dimensional guided biplane Simpson's method of disc measurements by echocardiography. Echocardiography was performed before implantation and at 2-year intervals thereafter in the absence of clinical events or at the onset of HF.

\section{Definition}

Progressive TR was defined as increased TR grade of [?]2 degrees and/or TRPG of $>30 \mathrm{mmHg}$ after implant, and TRPG of $>30 \mathrm{mmHg}$ was suggestive of possible pulmonary hypertension ${ }^{9}$. Moderate TR (grade III) was defined as a regurgitant jet extending to less than half of the right atrium, whereas severe TR (grade IV) as a jet extending to more than half of the length of the right atrium ${ }^{10}$. HF hospitalization was defined as the occurrence of HF events according to a New York Heart Association functional class of III or IV in the absence of other alternative diagnoses. HF symptoms were classified as the New York Heart Association functional class II-IV required medical treatment. Cardiovascular mortality was defined as sudden death related to arrhythmias, HF, and myocardial infarction. All-cause mortality was defined as death related to any cause, such as sudden death with undefined reasons, natural course, sepsis, malignancy, and cardiovascular disease.

\section{Study end-points}

The primary study endpoint was TR progression (TR grade [?]3) and/or abnormal TRPG levels (PG >30 $\mathrm{mmHg}$ ). The secondary study end-points were late-onset atrial fibrillation, HF hospitalization, sudden death or ventricular tachyarrhythmias, cardiovascular mortality, and all-cause mortality.

\section{Statistical analysis}

Data are presented as mean +- standard deviation or numbers (percentages). Clinical characteristics of the study groups were compared using the $t$-test for continuous variables and Chi-square test for categorical variables. Kaplan-Meier curve analysis was performed with the log-rank test for HF hospitalization and progressive TR in both groups during the follow-up period. Univariate and multivariate Cox regression analyses for HF hospitalization and progressive TR were performed to determine significant determinants. Multivariate Cox regression analysis included a hazard ratio $(\mathrm{HR})<0.100$ for HF hospitalization and progressive TR in univariate Cox regression analyses. Statistical analysis was performed using statistical software (SPSS for Windows, Version 22), and a two-sided $p$-value of $<0.05$ indicated statistical significance.

\section{Results}

\section{Baseline characteristics of the study patients}

Baseline characteristics of the study participants are listed in Table 1. During a median 4.9 (4.7-5.1) years follow-up, 198 (18.4 \%) patients (group 1, mean age $72.1+-9$ years; $59.6 \%$ female) experienced progressive post-implant TR, whereas 877 patients (group 2, mean age $71.9+-12$ years; $49.8 \%$ female) did not have progressive post-implant TR. The percentage of female individuals was higher in group 1 than group 2 . Additionally, the prevalence of atrial fibrillation (paroxysmal or non-paroxysmal) was also higher in group 1. A higher percentage of sick sinus syndrome for PPM was noted in group 1 (group 1 vs. group 2; 63.1\% vs. $53.9 \% ; \mathrm{p}=0.022$ ). There was no difference in the distribution of ventricular lead position, pacing QRS duration, ventricular pacing percentage, serum creatinine level and medication used between the 2 groups.

\section{Pre-implant and post-implant echocardiographic parameters of study patients}

At pre-implant, group 1 had significantly larger LA dimension (group 1 vs. group 2; $37.8+-6.6 \mathrm{~mm}$ vs. 36.7 $+-6.3 \mathrm{~mm} ; \mathrm{p}=0.063$ ) and significantly higher average TRPG (group 1 vs. group $2 ; 23.1+-4.9 \mathrm{mmHg}$ vs. $20.7+-6.1 \mathrm{mmHg} ; \mathrm{p}<0.001$ ) than group 1 (Table 2 ). The two groups did not differ in LVEDV and LVEF.

The median follow-up period was similar between the two groups (group 1 vs. group $2 ; 4.7$ (4.4-5.4) years vs. $4.5(4.2-4.8)$ years; $\mathrm{p}=0.610)$. At post-implant, group 1 had significantly larger LA dimension, lower 
LVEF and more severe TR grade than group 2. Additionally, group 1 had significantly higher post-implant TRPG (group 1 vs. group 2; $35.9+-9.1 \mathrm{mmHg}$ vs. $21.8+-5.4 \mathrm{mmHg} ; \mathrm{p}<0.001$ ) and larger changes in post-implant TRPG (group 1 vs. group 2; $12.8+-9.6 \mathrm{mmHg}$ vs. $1.1+-7.6 \mathrm{mmHg}$; $\mathrm{p}<0.001$ ) than group 2. Figure 2 showed the changes in pre-implant and post-implant TRPG in group $1(\mathrm{p}<0.001)$.

Figure 3 shows the cumulative incident rate of progressive TR grade and/or abnormal TRPG from $1.3 \%$ in the first year to $18.4 \%$ in the sixth year in the study cohort.

\section{Univariate and multivariate Cox regression analyses of predictors of progressive post-implant TR}

Female gender, higher pre-implant TRPG, larger post-implant LA dimension, lower post-implant LVEF and post-implant LVEF $<40$ were significant preditors of progressive post-implant TR (Table 3). However, only pre-implant TRPG (HR: 1.075; 95\% confidence interval $(\mathrm{CI}): 1.032-1.121 ; \mathrm{p}=0.001$ ) and post-implant LA dimension (HR: 1.076; 95\% CI: 1.038-1.114; p < 0.001) were independent predictors of progressive postimplant TR (Table 3).

\section{Clinical outcomes of the study patients}

During the follow-up period, group 1 had a significantly higher incidence of HF hospitalization compared to group $2(13.6 \%$ vs. $4.7 \% ; \mathrm{p}<0.001)$ (Table 4 and Figure 4$)$. However, the incidence of late-onset atrial fibrillation, sudden death or ventricular tachyarrhythmias, cardiovascular mortality, and all-cause mortality did not differ between the two groups (Table 4).

\section{Univariate and multivariate Cox regression analyses of predictors of $\mathrm{HF}$ hospitalization}

By univariate Cox regression analyses, older age, high body mass index, diabetes mellitus (DM), coronary artery disease (CAD), longer pacing QRS length, ventricular lead position at the lower septum and apex, larger pre-implant LA dimension, larger pre-implant LVEDV, larger post-implant LA dimension, larger post-implant LVEDV, lower post-implant LVEF, post-implant LVEF $<40 \%$, and progressive post-implant TR were significant preditors of HF hospitalization (Table 5). However, by multivariate Cox regression analyses, only older age (HR: 1.073; 95\% CI: 1.037-1.110; $\mathrm{p}<0.001$ ), CKD stage of $>3$ (moderate to severe CKD) (HR: 1.865; 95\% CI: 1.008-3.450; $\mathrm{p}=0.047$ ), and larger post-implant LVEDV (HR: 1.010; 95\% CI: $1.004-1.017 ; \mathrm{p}=0.001)$ were independently associated with HF hospitalization. Post-implant LVEF (HR: 0.957; 95\% CI: 0.934-0.980; $\mathrm{p}<0.001$ ) was independently inversely associated with HF hospitalization. Progressive post-implant TR (HR: 1.694; 95\% CI: 0.959-2.994; $\mathrm{p}=0.070$ ) had a non-significant trend toward HF hospitalization.

\section{Discussion}

In the present study, the cumulative rate of progressive TR ranged from $1.3 \%$ in the first year to $18.4 \%$ in the sixth year. Higher pre-implant TRPG and larger post-implant LA dimension were positively associated with progressive post-implant TR, which was associated with a trend toward HF hospitalization.

TR occurs mainly due to annular dilation and right ventricular enlargement, often secondary to LV dysfunction from myocardial or valvular causes, right ventricular volume and pressure overload, and cardiac chamber dilations ${ }^{11}$. Lead-related TR is an underdetermined problem and may be caused by lead-related tricuspid leaflet injury or perforation or lead entanglement, impingement, or adherence to the tricuspid valve 6 . However, lead-related tricuspid valve injury could not be fully detected and was only observed in $12 \%$ of patients with PPM-related severe TR by transthoracic echocardiography ${ }^{6}$. Kim et al. reported that abnormal TR developed in $21.2 \%$, worsened TR by [?] 1 grade in $24.2 \%$, and progressed to severe TR in $3.9 \%$ of patients with initially normal $\mathrm{TR}^{4}$. However, Al-Bawardy et al. reported a small but significant increase in the prevalence of moderate and severe TR, both acutely and chronically after a cardiac device implantation $^{5}$. Arabi et al. reported that TR was worsened by 1 grade in $70.8 \%$ and 2 grades in $17.1 \%$ of patients, and $19.5 \%$ of patients without baseline TR developed new-onset TR after the lead implantation in the follow-up period ${ }^{12}$. In this study, the cumulative rate of progressive post-implant TR (increased TR 
grade of [?]2 degrees and/or TRPG of $>30 \mathrm{mmHg}$ ) was from $1.3 \%$ in the first year to $18.4 \%$ in the sixth year. Moreover, higher pre-implant TRPG and larger post-implant LA dimension were independent predictors of progressive post-implant TR. Pacing-induced electrical and mechanical dyssynchrony of LV can also result in TR and $\mathrm{MR}^{13}$. However, in this study, pacing percentage and pacing QRS length was not associated with the development of progressive post-implant TR. In this study, larger post-implant LA size was an independent predictor of progressive post-implant TR. Our previous study showed that right and left atrial sizes were larger in patients with atrioventricular dyssynchrony after pacing ${ }^{14}$. Atrial enlargement is a well known predictor of atrial fibrillation. Utsunomiya et al reported that functional TR with a structurally normal tricuspid valve may occur secondary to chronic atrial fibrillation and is associated with advanced age and right atrial enlargement ${ }^{15}$.

In one small retrospective cohort study, significant lead-induced TR was associated with a significantly increased incidence of all-cause mortality and HF events in patients after PPM implantation ${ }^{16}$. Other studies also reported post-implant TR to be an independent risk factor for late death ${ }^{5,13}$. However, a significant proportion of patients in previous studies included patients with HF and receiving ICD and cardiac resynchronization therapy (CRT). Patients with ICDs and/or CRT devices usually have poor LVEF and advanced HF and consequently, higher incident HF hospitalization and mortality. In our study, we only enrolled patients receiving PPM implantation and excluded patients receiving ICD or CRT and those with prior history of HF, valvular heart disease and preexisting abnormal (mild-moderate, moderate or severe) TR and abnormal $(>30 \mathrm{mmHg}$ ) TRPG. In this large cohort study, progressive post-implant TR was significantly associated with HF hospitalization in univariate analysis and was associated with a non-significant trend toward HF hospitalization $(\mathrm{p}=0.070)$ in multivariate analysis (Table 5), and progressive post-implant TR was not associated with cardiovascular and all-cause mortality. Therefore, patients with preserved LV function and without valve disease underwent transvenous ventricular-based pacemaker implantation should have baseline echocardiography evaluation before implant and those with higher pre-implant TRPG should have more vigorously echocardiographic follow-up for the development of progressive post-implant TR.

\section{Study limitations}

One limitation of this study is its retrospective nature, including data from only one medical center. Because of older age, the all-cause mortality rate was relatively high in this study. Another limitation was the absence of baseline and follow-up right heart size and function by echocardiography. However, we still provided important information about lead-related post-implant TR progression and its associated outcomes in patients with transvenous ventricular-based PPM.

\section{Conclusions}

After a transvenous ventricular-based PPM implantation, $18.4 \%$ of patients experienced progressive postimplant TR and/or elevated TRPG. Patients with progressive post-implant TR had a higher incidence of HF hospitalization. Higher pre-implant TRPG and larger post-implant LA dimension were independent predictors of progressive post-implant TR.

\section{References}

1. Furman S, Schwedel J. An intracardiac pacemaker for Stokes-Adams seizures. N Engl J Med 1959;261:943-8.

2. Akerstrom F, Pachon M, Puchol A, et al. Chronic right ventricular apical pacing: adverse effects and current therapeutic strategies to minimize them. Int J Cardiol. 2014;15;173(3):351-60.

3. Paniagua D, Aldrich HR, Lieberman EH, Lamas GA, Agatston AS. Increased prevalence of significant tricuspid regurgitation in patients with transvenous pacemakers leads. Am J Cardiol. 1998;1;82(9):1130-2, A9.

4. Kim JB, Spevack DM, Tunick PA, et al. The effect of transvenous pacemaker and implantable cardioverter defibrillator lead placement on tricuspid valve function: an observational study. J Am Soc Echocardiogr. 2008;21(3):284-7. 
5. Al-Bawardy R, Krishnaswamy A, Rajeswaran J, et al. Tricuspid regurgitation and implantable devices. Pacing Clin Electrophysiol. 2015;38(2):259-66.

6. Lin G, Nishimura RA, Connolly HM, Dearani JA, Sundt TM 3rd, Hayes DL. Severe symptomatic tricuspid valve regurgitation due to permanent pacemaker or implantable cardioverter-defibrillator leads. J Am Coll Cardiol. 2005;17;45(10):1672-5.

7. Roeffel S, Bracke F, Meijer A, et al. Transesophageal echocardiographic evaluation of tricuspid valve regurgitation during pacemaker and implantable cardioverter defibrillator lead extraction. Pacing Clin Electrophysiol. 2002;25(11):1583-6.

8. Webster G, Margossian R, Alexander ME, et al. Impact of transvenous ventricular pacing leads on tricuspid regurgitation in pediatric and congenital heart disease patients. J Interv Card Electrophysiol. $2008 ; 21(1): 65-8$.

9. Ciurzyński M, Bienias P, Irzyk K, et al. Usefulness of echocardiography in the identification of an excessive increase in pulmonary arterial pressure in patients with systemic sclerosis. Kardiol Pol. 2011;69(1):9-15.

10. Polewczyk A, Kutarski A, Tomaszewski A, et al. Lead dependent tricuspid dysfunction: Analysis of the mechanism and management in patients referred for transvenous lead extraction. Cardiol J. 2013;20(4):402-10.

11. Rogers JH, Bolling SF. The tricuspid valve: current perspective and evolving management of tricuspid regurgitation. Circulation. 2009;26;119(20):2718-25.

12. Arabi P, Özer N, Ateş AH, Yorgun H, Oto A, Aytemir K. Effects of pacemaker and implantable cardioverter defibrillator electrodes on tricuspid regurgitation and right sided heart functions. Cardiol J. 2015;22(6):637-44.

13. Höke U, Auger D, Thijssen J, et al. Significant lead-induced tricuspid regurgitation is associated with poor prognosis at long-term follow-up. Heart. 2014;100(12):960-8.

14. Alizadeh A, Sanati HR, Haji-Karimi M, et al. Induction and aggravation of atrioventricular valve regurgitation in the course of chronic right ventricular apical pacing. Europace. 2011;13(11):1587-90.

15. Lin YS, Guo GB, Chen YL, et al. Atrial enlargement in symptomatic heart block patients with preserved left ventricular function: possibly related to atrioventricular dyssynchrony. Int J Cardiol. $2011 ; 5 ; 148(3): 280-4$.

\section{Figure legends}

Figure 1. Flowchart of the study enrollment.

Abbreviations: HF: heart failure; LVEF: left ventricular ejection fraction; DCM: dilated cardiomyopathy; TR: tricuspid regurgitation; TRPG: tricuspid regurgitation pressure gradient; TV: tricuspid valve.

Figure 2. Changes of the tricuspid regurgitation pressure gradient in group 1.

In group 1, the post-implant TRPG was significantly higher than pre-implant TRPG $(\mathrm{p}<0.001)$.

Figure 3. The cumulative incident rate of progressive post-implant tricuspid regurgitation.

The cumulative rate of progressive post-implant TR increased from $1.3 \%$ in the first year to $18.4 \%$ in the sixth year.

Figure 4. A Kaplan-Meier curve analysis for heart failure hospitalization.

Group 1 (with progressive post-implant tricuspid regurgitation and/or elevated tricuspid regurgitation pressure gradient) had a significantly higher incidence of heart failure hospitalization compared to group 2 (without progressive post-implant tricuspid regurgitation and/or elevated tricuspid regurgitation pressure gradient) (log-rank $\mathrm{P}<0.001)$.

\section{Hosted file}

Table 1.docx available at https://authorea.com/users/310064/articles/462484-progressivetricuspid-regurgitation-and-elevated-tricuspid-regurgitation-pressure-gradient-after- 
transvenous-permanent-pacemaker-implantation

\title{
Hosted file
}

Table 2.docx available at https://authorea.com/users/310064/articles/462484-progressivetricuspid-regurgitation-and-elevated-tricuspid-regurgitation-pressure-gradient-aftertransvenous-permanent-pacemaker-implantation

\section{Hosted file}

Table 3.docx available at https://authorea.com/users/310064/articles/462484-progressivetricuspid-regurgitation-and-elevated-tricuspid-regurgitation-pressure-gradient-aftertransvenous-permanent-pacemaker-implantation

\section{Hosted file}

Table 4.docx available at https://authorea.com/users/310064/articles/462484-progressivetricuspid-regurgitation-and-elevated-tricuspid-regurgitation-pressure-gradient-aftertransvenous-permanent-pacemaker-implantation

\section{Hosted file}

Table 5.docx available at https://authorea.com/users/310064/articles/462484-progressivetricuspid-regurgitation-and-elevated-tricuspid-regurgitation-pressure-gradient-aftertransvenous-permanent-pacemaker-implantation

\begin{abstract}
A total of 1670 patients received single ventricular-based or dual chamber pacemaker implantation between 2003-2017 (without implantable cardioverter defibrillator and
\end{abstract} cardiac resynchronization therapy implantation)

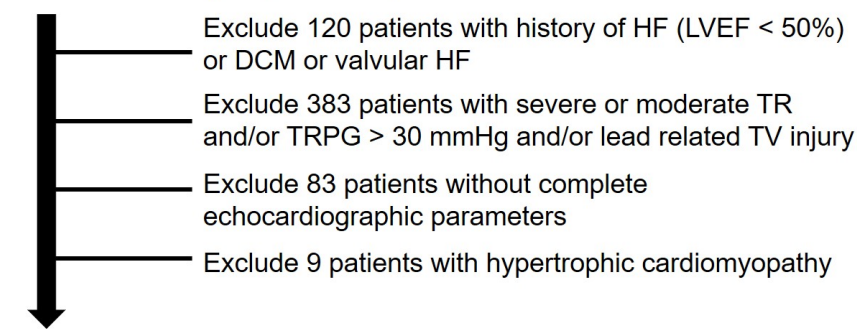

A total of 1075 patients were enrolled

$$
\begin{aligned}
& \text { Group } 1(\mathrm{~N}=198) \\
& \text { Increased degree of post- } \\
& \text { implant TR ( } \geq 2 \text { degrees) } \\
& \text { and/or abnormal TRPG }
\end{aligned}
$$




\section{The change of TRPG}
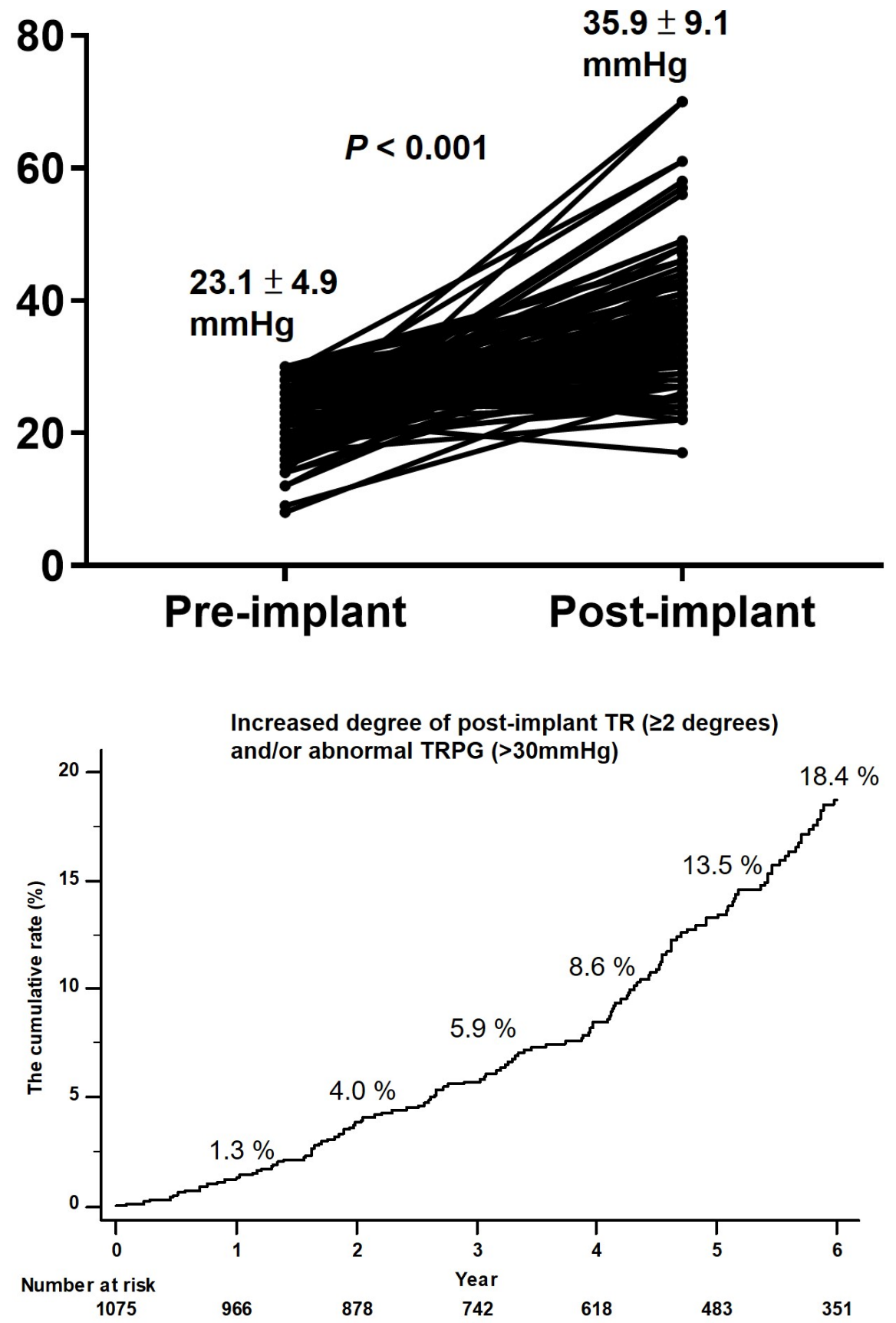


\section{HF hospitalization}

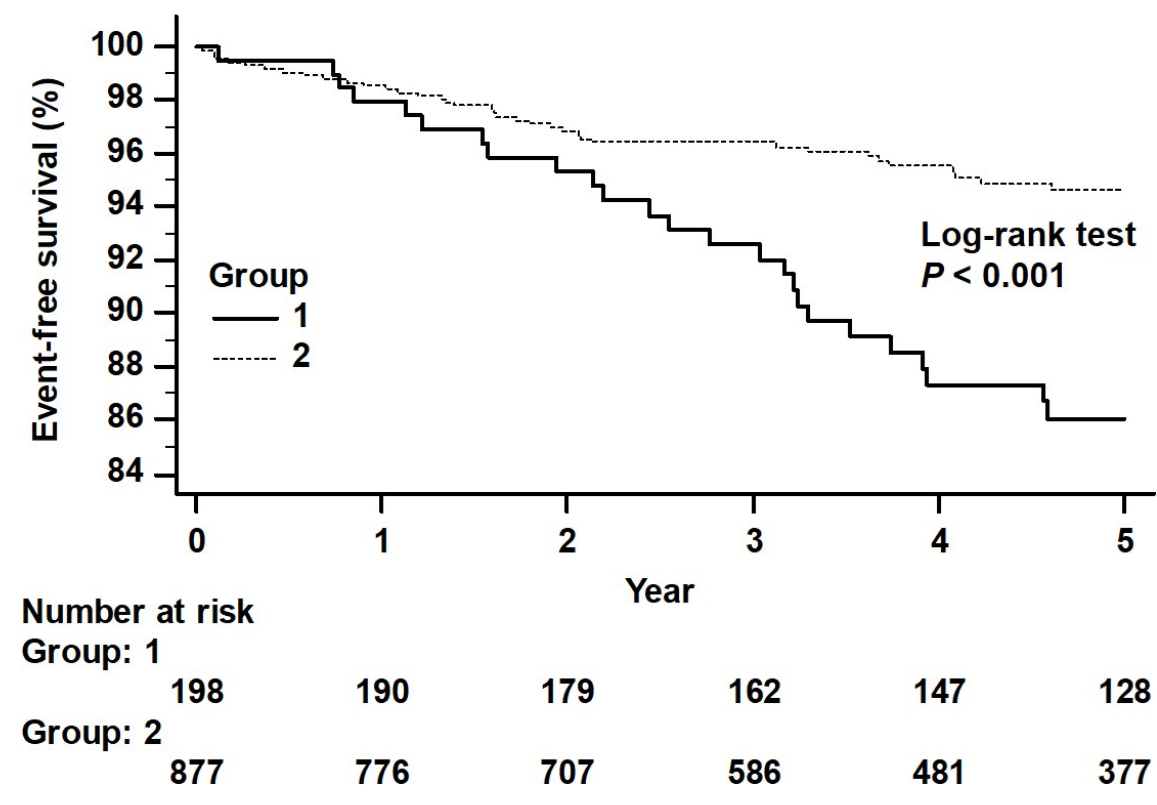

